\title{
Model Development of p-i-n Germanium Devices for Infrared Detection
}

\section{Caitlin R. Philippi ${ }^{*}$, John W. Zeller ${ }^{2}$, Nibir K. Dhar ${ }^{3}$, Priyalal Wijewarnasuriya ${ }^{4}$, Ashok K. Sood ${ }^{2}$, Harry Efstathiadis ${ }^{1}$}

\author{
${ }^{1}$ State University of New York Polytechnic Institute, Albany, NY, USA \\ ${ }^{2}$ Magnolia Optical Technologies Inc., Woburn, MA, USA \\ ${ }^{3}$ US Army Night Vision \& Electronic Sensors Directorate, Fort Belvoir, VA, USA \\ ${ }^{4}$ US Army Research Laboratory, Adelphi, MD, USA \\ Email: *crouse2@sunypoly.edu
}

How to cite this paper: Philippi, C.R., Zeller, J.W., Dhar, N.K., Wijewarnasuriya, P., Sood, A.K. and Efstathiadis, H. (2018) Model Development of p-i-n Germanium Devices for Infrared Detection. Optics and Photonics Journal, 8, 201-211.

https://doi.org/10.4236/opj.2018.86018

Received: May 16, 2018

Accepted: June 24, 2018

Published: June 27, 2018

Copyright $\odot 2018$ by authors and Scientific Research Publishing Inc. This work is licensed under the Creative Commons Attribution International License (CC BY 4.0).

http://creativecommons.org/licenses/by/4.0/ Open Access

\begin{abstract}
Germanium offers many benefits over groups III-V materials when used for infrared detection. Most importantly, germanium is compatible with Complementary Metal Oxide Semiconductor (CMOS) manufacturing which allows for a low-cost, high-throughput device, and it does not require cooling, which many III-V devices do. With the deposition of germanium directly on silicon, there will be a thermally induced strain due to the difference in thermal expansion coefficients between the two materials. When a biaxial tensile strain is present, the direct bandgap of germanium is lowered to $\sim 0.77 \mathrm{eV}$ and is capable of absorbing longer wavelengths. We have used a two-step deposition process to create a strained germanium film in order to fabricate a photodetector device. Our device has a dark current of $1.35 \mathrm{nA}$ and a photocurrent of $22.5 \mathrm{nA}$ at $1570 \mathrm{~nm}$ wavelength. Next, we developed a model in order to compare theoretical results with experimental results. The results indicate that the model is in close agreement with our measured data, and we are therefore able to use it to model future devices.
\end{abstract}

\section{Keywords}

Infrared Detection, Germanium, Photodetector, Model, TCAD

\section{Introduction}

Infrared detection has applications in a wide range of fields. Telecommunications has been vastly improved with the use of fiber optics due to decreased cost and increased data rates compared to using copper wire [1]. The capacity of a 
single fiber is over hundreds of $\mathrm{Tb} / \mathrm{s}$ [2]. There are also many military applications, including biochemical threat detection, night vision and the ability to image hostile mortar fire and muzzle flash [1].

Traditionally, groups III-V materials have been used to fabricate infrared detectors due to their high absorption efficiency and high carrier drift velocity [3]. However, fabrication of devices using these materials can be complex and expensive [1]. Groups III-V materials also are not compatible with Complementary Metal Oxide Semiconductor (CMOS) manufacturing since they act as contaminates in silicon. Additionally, devices fabricated from these materials may require cooling, some down to $77 \mathrm{~K}$ [4].

On the other hand, germanium, a group IV material, does not act as a contaminant in silicon and is compatible with CMOS manufacturing. This allows for a low-cost, high-throughput device. Germanium is also capable of achieving a relatively low dark current $(<1 \mu \mathrm{A})$ without cooling. However, germanium does have a few disadvantages, mainly that it has a $4.2 \%$ lattice mismatch with silicon and that it is predominantly an indirect bandgap material. The lattice mismatch between silicon and germanium can cause misfit dislocations at the interface between the two materials, which can degrade device performance [5]. Germanium is a poor absorber of infrared light due to the fact that it is an indirect bandgap material. The indirect bandgap is predominant at $0.66 \mathrm{eV}$; however, its direct bandgap is only $140 \mathrm{meV}$ above the indirect bandgap at $0.8 \mathrm{eV}$ [4]. With a $0.2 \%$ thermally induced strain, germanium can be shifted toward a direct bandgap material at $0.77 \mathrm{eV}$, which will allow for absorption of longer wavelengths [4]. This is due to the introduction of a biaxial tensile stain, and it extends the absorption range into the $1560-1620 \mathrm{~nm}$ wavelength range [6]. Using germanium in a $\mathrm{p}-\mathrm{i}-\mathrm{n}$ junction allows the depletion region width to be tailored simply by changing the thickness of the intrinsic layer [7]. This allows the optimization of quantum efficiency and frequency response.

It can be beneficial and more efficient to model devices using Technology Computer Aided Design (TCAD) before spending much time and resources on test wafer runs. Many groups have taken advantage of developing a model for their work [8] [9] [10]. Using TCAD allows us to simulate the fabrication of unique designs, materials and properties such as doping, thickness, etc. For these reasons, we have developed a model for a previously reported device [11] in order to compare experimental and theoretical results. This will allow us to translate the model for simulations of future device designs.

\section{Device Design and Fabrication}

We have successfully fabricated a germanium $\mathrm{p}-\mathrm{i}-\mathrm{n}$ infrared detector on a 300 $\mathrm{mm}$ p-type silicon wafer. Using a $300 \mathrm{~mm}$ wafer results in a higher yield, which can save time and expenses. We deposit pure germanium on the silicon wafer using a two-step growth process, which ensures a quality, smooth epitaxial germanium film without using very thick traditional SiGe buffer layers. We per- 
formed this two-step deposition using an Applied Materials Centura Reduced Pressure Chemical Vapor Deposition (RPCVD) system. After the film deposition, we then performed ion implantation and metallization to create front contacts to complete the device.

\subsection{Two-Step Growth Process}

Before we begin the deposition, we first perform an in-situ clean where we heat the silicon substrate to $\sim 1050^{\circ} \mathrm{C}$ for 1 - 2 minutes under hydrogen of ultra-high purity. This reduced the native oxide on the surface and leaves the silicon H2-terminated. For the first step of the deposition, we deposited a thin $(\sim 100$ $\mathrm{nm})$ p-type germanium buffer layer at a lower temperature $\left(\sim 325^{\circ} \mathrm{C}-425^{\circ} \mathrm{C}\right)$. The deposition of a buffer layer allows for a smooth film without islands. Usually germanium follows Stranski-Krastanov (or layer-plus-island) growth. However, at this low temperature, the island growth of germanium is kinetically suppressed and therefore will only grow as a layer [12]. Instead of forming islands, it is energetically favorable to form dislocations since an island has a higher surface energy. Below the critical thickness for germanium on silicon, which is $\sim 1 \mathrm{~nm}$, near epitaxial growth will occur. However, above this thickness, stresses due to the mismatched lattices can result in plastic deformation, which results in the formation of dislocations [13]. The dislocation concentration will increase until a minimum free energy of the system is reached [14]. If the stress in a material becomes too large to be accommodated by dislocations, cracks may form to release the strain [15]. Since the buffer layer is p-type, it also allows most of the dislocations to remain outside of the depletion region which improves device performance [16].

The second step involves germanium deposition of a thick $(\sim 1.5 \mu \mathrm{m})$ intrinsic germanium region. This layer is deposited at a higher temperature $\left(\sim 600^{\circ} \mathrm{C}\right)$ which allows for a better crystal quality as well as a higher growth rate, which is important due to its thickness.

\subsection{Remaining Device Fabrication}

Once the intrinsic germanium layer is deposited, the next step is to form a p-i-n junction. First, we deposited a layer of $\mathrm{SiO}_{2}$ directly on the i-Ge region. Next, we opened windows of $\sim 50 \mu \mathrm{m}$ and deposited a $20 \mathrm{~nm}$ screen oxide layer. We then performed ion implantation of phosphorus to form an $\mathrm{n}+$ region within the window. The screen oxide layer was then stripped, and a tantalum nitride (TaN) diffusion barrier layer was deposited. To form the contacts, we then deposited copper within the oxide windows and finished the device by performing chemical mechanical polishing (CMP). The fabrication steps as well as a schematic of the finished device can be found in Figure 1.

\section{Device Characterization}

In order to characterize the film quality and device performance, several differ- 
ent techniques were utilized. To confirm crystalline growth of germanium, we took Transmission Electron Microscope (TEM) images as well as Energy-Dispersive X-ray Spectroscopy (EDS) data. These can be seen in Figure 2. We also used Secondary Ion Mass Spectroscopy (SIMS) to measure the doping levels of the p-and n-regions of the junction, as well as to ensure that any background doping of the intrinsic region was not too high (usually $>10^{14}$ atoms $/ \mathrm{cm}^{3}$ ). The $\mathrm{n}-\mathrm{Ge}$ was measured at $5.5 \times 10^{18} \mathrm{~cm}^{-3}$, the $\mathrm{i}-\mathrm{Ge}$ was $5.0 \times 10^{14}$ $\mathrm{cm}^{-3}$, and the $\mathrm{p}$-Ge was $1.0 \times 10^{18} \mathrm{~cm}^{-3}$. Finally, Current-Voltage (I-V) measurements were performed in order to quantify the performance of the finished photodetector. Since this paper focuses on modelling the I-V measurements of this device, we shall focus on those data. For all other characterization data, please refer to [11].

I. p-type $<100>$ Si wafer

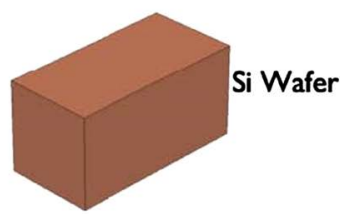

4. Ion implant to form n-type Ge

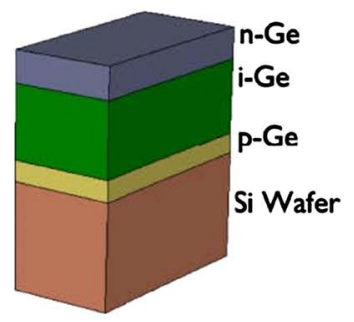

2. $\sim 100 \mathrm{~nm}$ p-Ge seed layer, $350-400^{\circ} \mathrm{C}$

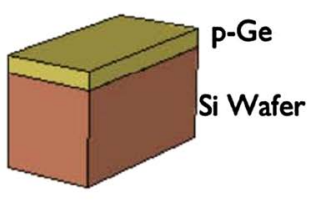

5. Deposit oxide

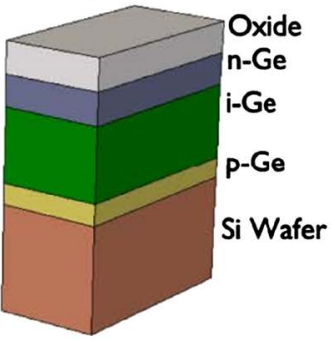

3. $\sim 1.5 \mu \mathrm{m}$ i-Ge layer, $550-600^{\circ} \mathrm{C}$

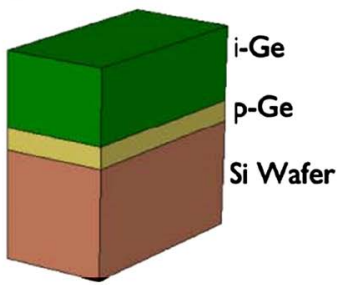

6. Deposit Cu contacts

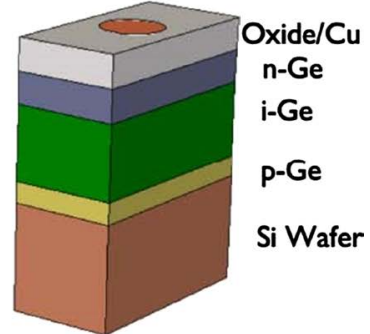

Figure 1. Schematic of the process flow for device fabrication. Final device shown in step six (not drawn to scale).

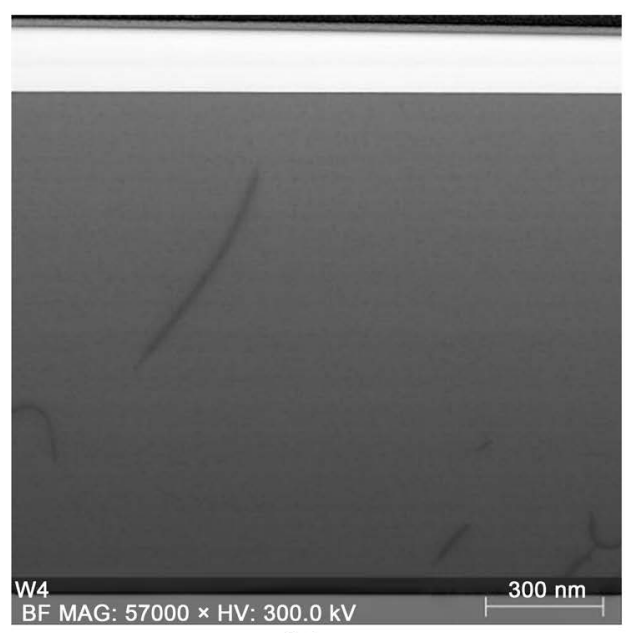

(a)

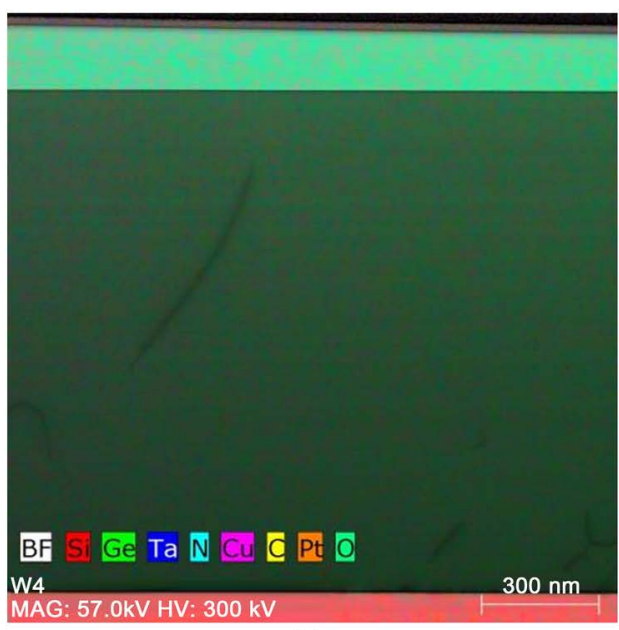

(b)

Figure 2. (a) TEM image of germanium film on silicon wafer; (b) EDS confirming germanium growth [16]. 


\subsection{Current-Voltage Measurements}

We performed I-V measurements using a two-probe station with a Keithley 2400 SourceMeter and a light source emitting within the infrared wavelength region. In this setup, one probe makes contact with the top copper contact on the device, while the other probe makes contact with the bottom of the device resting on the gold-plated stage.

Some important information we gather from I-V curves are the dark current and photocurrent at $-1 \mathrm{~V}$ and $+1 \mathrm{~V}$. From this, we can calculate the ratio between dark current and photocurrent in order to confirm that we are absorbing photons and generating and collecting carriers. We can also calculate the ratio between forward and reverse bias dark current to ensure there is diode behavior. Lastly, we look to see if the curve is flat under reverse bias. The flatter the curve, the less leakage current is present. A lower leakage current is indicative of a higher quality film; for example, a low defect density. Figure 3 shows an I-V curve measured from our fabricated device.

The dark current at $-1 \mathrm{~V}$ is $1.35 \mathrm{nA}$, which is low relative to other germanium devices in literature [17] [18] [19]. The photocurrent at $-1 \mathrm{~V}$ is $22.5 \mathrm{nA}$, giving a ratio between dark current and photocurrent of 16.7, which indicates photons with a wavelength of $1570 \mathrm{~nm}$ were absorbed and generated carriers were collected. At $+1 \mathrm{~V}$, the dark current was $0.15 \mathrm{~mA}$ as was the photocurrent. The ratio between the forward and reverse dark current is $1.11 \times 10^{5}$, exhibiting strong diode behavior. In the reverse bias region, the curve is flat which indicates there

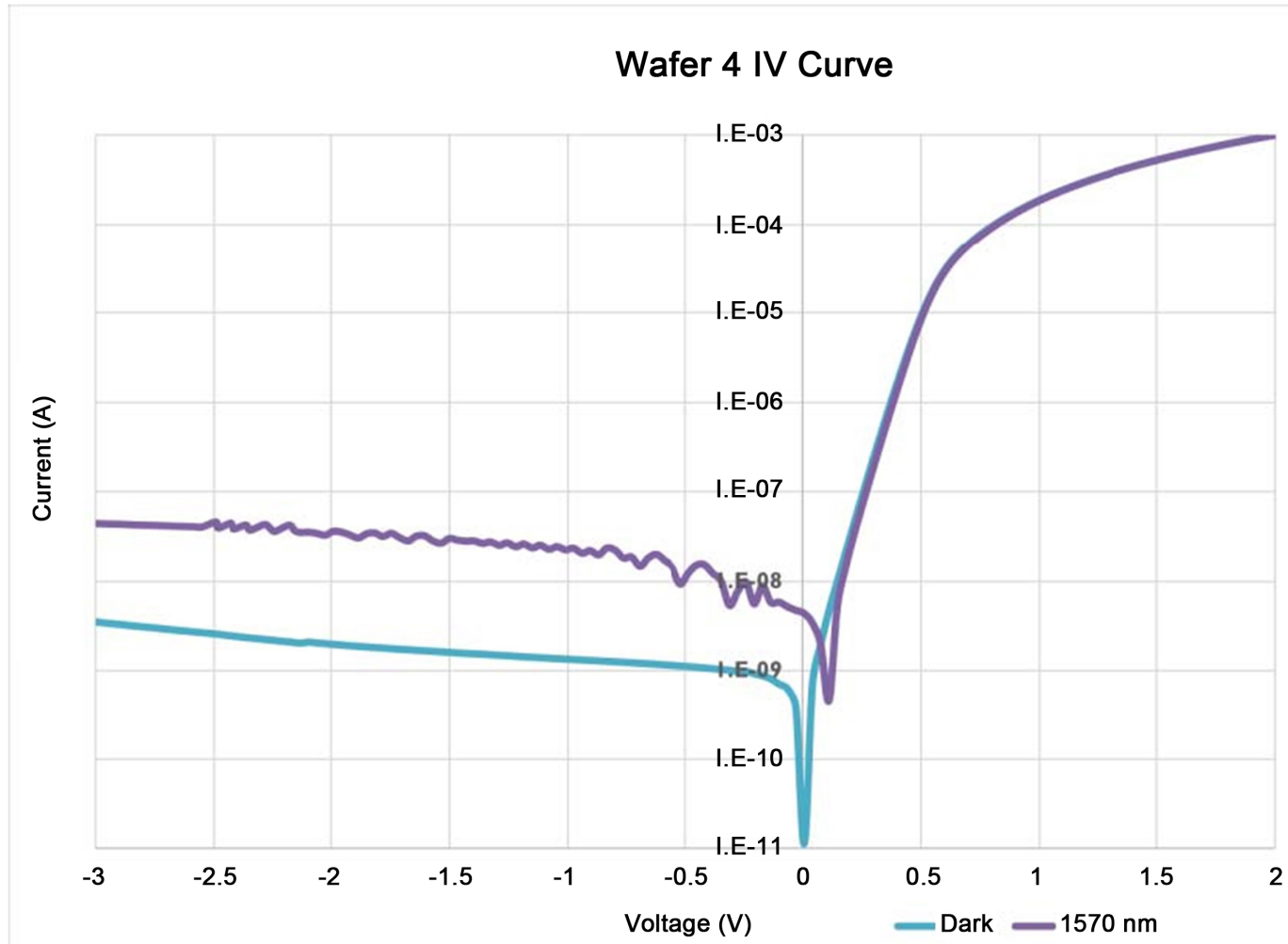

Figure 3. I-V curve showing dark current and photocurrent for our fabricated device. 
is a low leakage current and therefore a low defect density within the film.

\section{Device Modelling}

As previously mentioned, it can be beneficial to create a model for a device to be able to simulate the performance and/or characteristics of the device. This allows us to "test" different materials, structures and properties without requiring resources for device fabrication. Instead, we can perform simulations to help us find the optimum characteristics and then fabricate that device. To perform our simulations, we used Synopsys Sentaurus TCAD.

\subsection{Synopsys Sentaurus}

Sentaurus offers a suite of TCAD tools which allows engineers to simulate the fabrication, operation and reliability of semiconductor devices [20]. We used Sentaurus Process in order to emulate the fabrication of the device. This enables us to imitate film deposition, etching and annealing as well as defect formation and induced strain.

The next tool we use is Sentaurus Device, which allows us to simulate the electrical characteristics of the device such as dark current and photocurrent, quantum efficiency, mobility and more. Finally, Sentaurus Visual can be used to view the device structure and properties as well as create graphs for data such as $\mathrm{I}-\mathrm{V}$ curves. Sentaurus also includes many physical models for simulation. For our devices, three main models are important: mobility models, recombination models, and stress and strain models. These models represent the physical processes in the device.

\subsubsection{Mobility Models}

When considering the mobility models, we focus on the high field saturation model and the doping dependence model. The high field saturation model takes into account the fact that under high electric fields, the carrier drift velocity saturates instead of remaining proportional to the electric field. Since our device has a p-i-n junction, there will be a relatively high electric field and the carrier drift velocity will saturate. Equation (1) shows the formula used for mobility under a high electric field, which depends upon the saturation velocity of the carrier, $v_{\text {sat }}$ the low field mobility $\mu_{\text {low }}$ and a temperature dependent constant, $\beta$ [20].

$$
\mu(F)=\frac{(\alpha+1) \mu_{\text {low }}}{\alpha+\left[1+\left(\frac{(\alpha+1) \mu_{\text {low }} F_{h f s}}{v_{\text {sat }}}\right)^{\beta}\right]^{1 / \beta}}
$$

We also use the doping dependence model since we have doped germanium which makes up the $\mathrm{p}-\mathrm{i}-\mathrm{n}$ junction. This model uses the mobility degradation due to impurity scattering. Equation (2) expresses the doping-dependent mobility [20]. This mobility model depends upon the reference doping $\left(P_{c}\right)$ and carrier concentration $\left(N_{A}\right.$ and $\left.N_{D}\right)$. 


$$
\mu_{d o p}=\mu_{\min 1} \exp \left(\frac{P_{c}}{N_{A, 0}+N_{D, 0}}\right)+\frac{\mu_{c o n s t}-\mu_{\min 2}}{1+\left(\frac{N_{A, 0}+N_{D, 0}}{C_{t}}\right)^{\alpha}}-\frac{\mu_{1}}{1+\left(\frac{C_{s}}{N_{A, 0}+N_{D, 0}}\right)^{\beta}}
$$

\subsubsection{Recombination Models}

For our devices, we are mainly concerned with Shockley-Read-Hall (SRH) recombination and Auger recombination. SRH recombination occurs when an electron and hole recombine through a defect within the bandgap of the material. This is of special concern because our film contains defects which act as trap states and can increase SRH recombination. As seen in Equation (3), SRH recombination depends on the energy level of the trap state $\left(E_{\text {trap }}\right)$, the carrier concentrations, $n$ and $p$, as well as the carrier lifetime, $\tau$, for electrons and holes $[20]$.

$$
R_{n e t}^{S R H}=\frac{n p-n_{i, e f f}^{2}}{\tau_{p}\left(n+n_{1}\right)+\tau_{n}\left(p+p_{1}\right)}
$$

Auger recombination involves three carriers. When an electron recombines, instead of emitting a photon, the energy is transferred to another electron which will eventually relax back to the band edge. It is most prevalent at high carrier concentrations caused by heavy doping. Again, we have areas of high doping to form our p-i-n junction, so Auger recombination must also be considered. Equation (4) shows how Auger recombination depends on the carrier concentration of the doped material ( $n$ and $p$ ) [20].

$$
R_{n e t}^{A}=\left(C_{n} n+C_{p} p\right)\left(n p-n_{i, e f f}^{2}\right)
$$

\subsubsection{Stress and Strain Models}

Stress and strain play a very important role in terms of our germanium film characteristics and quality. The strain is important because it will alter the bandgap of germanium, allowing absorption of longer wavelengths.

These models make use of the deformation potentials. This is the effective electric potential experienced by free electrons in a semiconductor resulting from a local deformation in the crystal lattice. If strain is present, there will be a deformation in the crystal lattice and the electrons will experience a different electric potential, causing the bandgap to change. The change in energy for the valance band and conduction band, which depends on the deformation potential $\left(\Xi_{d}\right.$ ) can be found in Equation (5) [20]. The deformation potential is the effective electric potential experienced by free electrons in a semiconductor resulting from a local deformation in the crystal lattice, in other terms, an induced strain. The overall change in energy, which is dependent upon the induced strain, $(\varepsilon)$ is shown in Equation (6) [20]. $\varepsilon_{x x}$ corresponds to the components of the strain tensor in the crystal coordinate system.

$$
\begin{aligned}
& \Delta E_{V, i}=a\left(\varepsilon_{11}^{\prime}+\varepsilon_{22}^{\prime}+\varepsilon_{33}^{\prime}\right) \pm \delta E \\
& \Delta E_{C, i}=\Xi_{d}\left(\varepsilon_{11}^{\prime}+\varepsilon_{22}^{\prime}+\varepsilon_{33}^{\prime}\right)+\Xi_{u} \varepsilon_{i i}^{\prime}
\end{aligned}
$$




$$
\delta E=\sqrt{\frac{b^{2}}{2}\left(\left(\varepsilon_{11}^{\prime}-\varepsilon_{22}^{\prime}\right)^{2}+\left(\varepsilon_{22}^{\prime}-\varepsilon_{33}^{\prime}\right)^{2}+\left(\varepsilon_{11}^{\prime}-\varepsilon_{33}^{\prime}\right)^{2}\right)+d^{2}\left(\varepsilon_{12}^{\prime 2}+\varepsilon_{13}^{\prime 2}+\varepsilon_{23}^{\prime 2}\right)}
$$

\section{Results of Simulation and Discussion}

We have developed a model for the device we fabricated and characterized shown in previous sections and in [11]. Using Sentaurus Process, we were able to emulate strained germanium deposition, ion implantation of phosphorus and contact deposition. The dark current and photocurrent were simulated using Sentaurus Device. Using Sentaurus Visual, we were able to plot I-V curves in order to compare them with the experimental data to confirm a successful model. Figure 4 shows the experimental and theoretical curves plotted together.

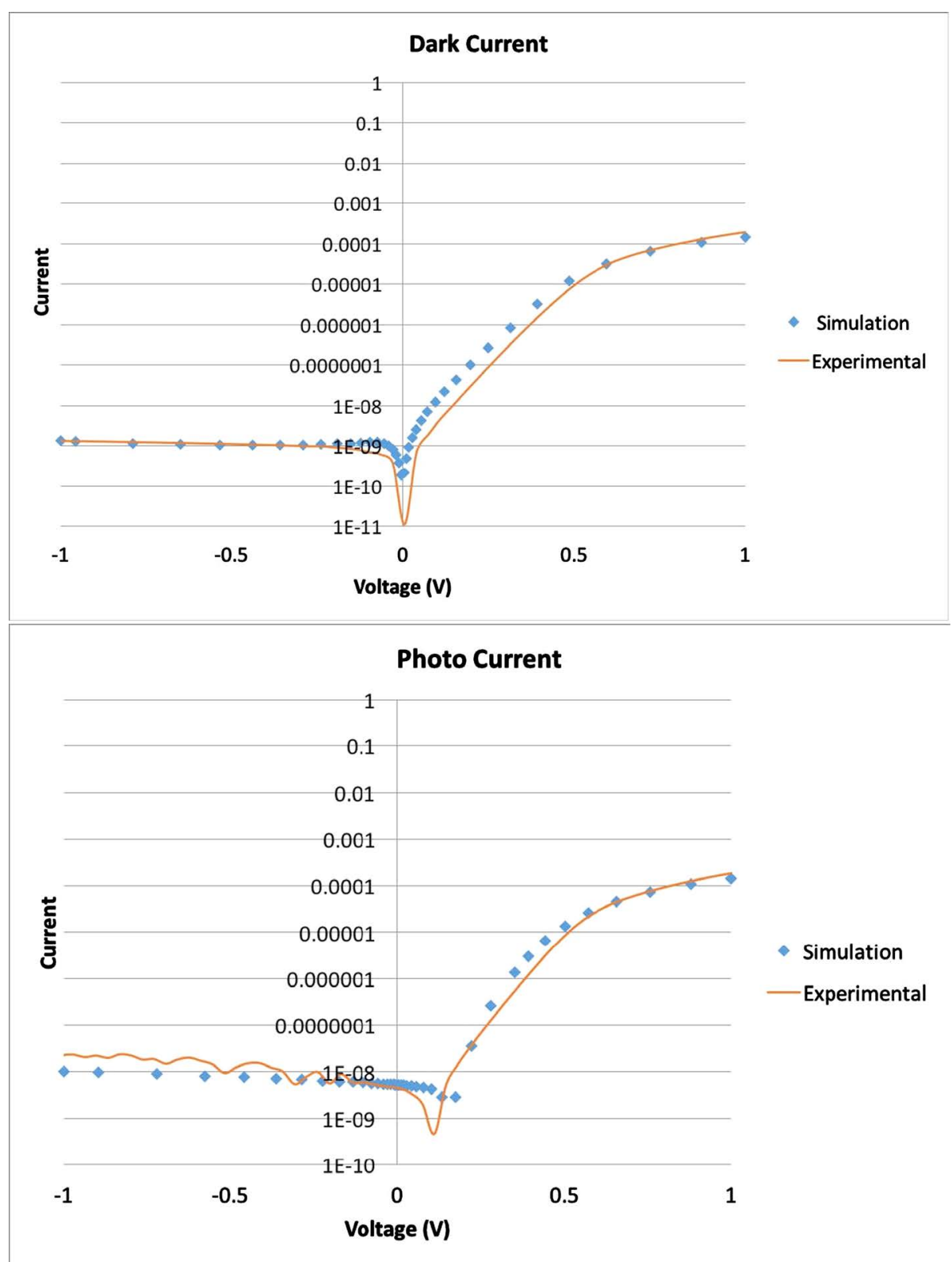

Figure 4. Simulated and experimental I-V curves for dark current and photocurrent. 
Table 1. Simulated and experimental data.

\begin{tabular}{ccccccc}
\hline $\begin{array}{c}\text { Simulated vs. } \\
\text { Experimental }\end{array}$ & $\begin{array}{c}\text { Dark at } \\
-1 \mathrm{~V}(\mathrm{nA})\end{array}$ & $\begin{array}{c}\text { Photo at } \\
-1 \mathrm{~V}(\mathrm{nA})\end{array}$ & $\begin{array}{c}\text { Dark at } \\
+1 \mathrm{~V}(\mathrm{~mA})\end{array}$ & $\begin{array}{c}\text { Photo at } \\
+1 \mathrm{~V}(\mathrm{~mA})\end{array}$ & $\begin{array}{c}\text { Photo: Dark } \\
\text { Ratio }\end{array}$ & $\begin{array}{c}\text { Forward: } \\
\text { Reverse Ratio }\end{array}$ \\
\hline Simulated & 1.353 & 10.2 & 0.15 & 0.15 & 7.54 & $9.02 \times 10^{6}$ \\
Experimental & 1.35 & 22.5 & 0.2 & 0.2 & 16.7 & $6.75 \times 10^{6}$ \\
\hline
\end{tabular}

This model is in close agreement with experimental results concerning the areas we are interested in. As discussed earlier, we look at the dark current at -1 $\mathrm{V}$, the ratio between the dark current and photocurrent at $-1 \mathrm{~V}$, and the ratio between forward and reverse dark current. We also look to see if the dark current curve is flat under reverse bias to ensure minimum leakage current. As seen in Table 1, these data are in overall agreement. There is a slight difference between experimental and theoretical photocurrent, which we attribute to the fact that the incident optical power had to be estimated for the simulation. There is also a fluctuation in the I-V curve for experimental photocurrent. We believe this is due to the physical limitations of our testing platform. The general behavior and function of the device, most importantly in terms of the ratios, allow us to accept this model as accurate for our devices and purposes.

\section{Conclusion and Future Work}

Germanium is a low-cost alternative to traditional III-V group materials and is compatible with CMOS manufacturing. We have successfully fabricated and tested a functioning $\mathrm{p}-\mathrm{i}-\mathrm{n}$ germanium infrared detector. We have measured a relatively low dark current at $1.35 \mathrm{nA}$ and a photocurrent at $1570 \mathrm{~nm}$ wavelength of $22.5 \mathrm{nA}$, indicating that we are absorbing photons and generating carriers. We were able to develop a model for our device which is in agreement with experimental results. Now that we have an accurate model for germanium $\mathrm{p}-\mathrm{i}-\mathrm{n}$ detectors, we will be able to use this as a basis in order to model other unique devices in the future.

\section{Acknowledgements}

This work was carried out under DARPA Phase II SBIR Program. We would like to thank Dr. Whitney Mason DARPA/MTO, Ms. Susan Celis, Director, Small Business Program Office and Mr. Oscar Cerna of DARPA/SBIR for their help and support. The views, opinion, and/or findings expressed are those of the authors and should not be interpreted as representing the official views or policies of the Department of Defense or the US Government.

\section{References}

[1] Sood, A.K., Zeller, J.W., Richwine, R.A., Puri, Y.R., Efstathiadis, H., Haldar, P., Dhar, N.K. and Polla, D.L. (2015) SiGe Based Visible-NIR Photodetector Technology for Optoelectronic Applications. In: Yasin, M., et al., Eds., Advances in Optical Fiber Technology. Fundamental Optical Phenomena and Applications, InTech Publications, Chicago. 
[2] Agrell, E., Karlsson, M., Chraplyvy, A.R., Richardson, D.J., Krummrich, P.M., Winzer, P., Roberts, K., Fischer, J.K., Savory, S.J. and Eggleton, B.J. (2016) Roadmap of Optical Communications. Journal of Optics, 18, 1-40. https://doi.org/10.1088/2040-8978/18/6/063002

[3] LaPierre, R.R., Robson, M., Azizur-Rahman, K.M. and Kuyanov, P. (2017) A Review of III-V Nanowire Infrared Photodetectors and Sensors. Journal of Physics D: Applied Physics, 50, 1-10. https://doi.org/10.1088/1361-6463/aa5ab3

[4] Wang, J. and Lee, S. (2011) Ge-Photodetectors for Si-Based Optoelectronic Integration. Sensors, 11, 696-718. https://doi.org/10.3390/s110100696

[5] Gersten, J.I. and Smith, F.W. (2011) The Physics and Chemistry of Materials. John Wiley \& Sons, Inc., New York.

[6] Wada, K. and Liu, J. (2003) Strain-Induced Band Gap Shrinkage in Ge Grown on Si Substrate. Applied Physics Letters, 82, 2044-2046. https://doi.org/10.1063/1.1564868

[7] Sze, S.M. and Ng, K.K. (2007) Physics of Semiconductor Devices. 3rd Edition, John Wiley \& Sons, Inc., New York.

[8] Lee, J.-M., Cho, S.-H. and Choi, W.-Y. (2016) An Equivalent Circuit Model for a Ge Waveguide Photodetector on Si. IEEE Photonics Technology Letters, 28, 2435-2438. https://doi.org/10.1109/LPT.2016.2598369

[9] Masini, G., Colace, L., Assanto, G., Luan, H.-C. and Kimerling, L.C. (2001) High-Performance p-i-n Ge on Si Photodetectors for the Near Infrared: From Model to Demonstration. IEEE Transactions on Electron Devices, 48, 1092-1096. https://doi.org/10.1109/16.925232

[10] Peschka, D., Thomas, M., Glitzky, A., Nurnberg, R., Gartner, K., Virgilio, M., Guha, S., Schroeder, T., Capellini, G. and Koprucki, T. (2015) Modeling of Edge-Emitting Lasers Based on Tensile Strained Germanium Microstrips. IEEE Photonics Journal, 7, Article ID: 1502115. https://doi.org/10.1109/JPHOT.2015.2427093

[11] Rouse, C., Zeller, J.W., Efstathiadis, H., Haldar, P., Lewis, J.S., Dhar, N.K., Wijewarnasuriya, P., Puri, Y.R. and Sood, A.K. (2016) Development of Low Dark Current SiGe Near-Infrared PIN Photodetectors on $300 \mathrm{~mm}$ Silicon Wafers. Optics and Photonics, 6, 61-68. https://doi.org/10.4236/opj.2016.65009

[12] Michel, J., Liu, J. and Kimerling, L.C. (2010) High-Performance Ge-on-Si Photodetectors. Nature Photonics, 4, 527-534. https://doi.org/10.1038/nphoton.2010.157

[13] Washburn, J., Kvam, E.P. and Liliental-Weber, Z. (1991) Defect Formation in Epitaxial Crystal Growth. Journal of Electronic Materials, 20, 155-171. https://doi.org/10.1007/BF02653317

[14] Lufaso, M. (2018) Solid State Chemistry. https://www.unf.edu/ michael.lufaso/chem4627/ch5_solid_state.pdf

[15] Grundmann, M. (2016) The Physics of Semiconductors: An Introduction Including Nanophysics and Applications. 3rd Edition, Springer, New York. https://doi.org/10.1007/978-3-319-23880-7

[16] Sood, A.K. and Zeller, J.W. (2017) SiGe Focal Plane Array Detector Technology for Near-Infrared Imaging. International Journal of Engineering Research and Technology, 10, 81-103.

[17] Chen, H.T., Verheyn, P., De Heyn, P., Lepage, G., De Coster, J., Absil, P., Roelkens, G. and Van Campenhout, J. (2015) High-Responsivity Low-Voltage 28-Gb/s Ge p-i-n Photodetector with Silicon Contacts. Journal of Lightwave Technology, 33, 820-824. https://doi.org/10.1109/JLT.2014.2367134

[18] Lin, Y., Lee, H., Bao, S., Guo, X., Wang, H., Michel, J. and Tan, C.S. (2017) 
High-Efficiency Normal-Incidence Vertical p-i-n Photodetectors on a Germanium-on-Insulator Platform. Photonics Research, 5, 702-709.

https://doi.org/10.1364/PRJ.5.000702

[19] Chen, G., Yu, Y., Xio, X. and Zhang, X. (2016) High Speed and High Power Polarization Insensitive Germanium Photodetector with Lumped Structure. Optics EXpress, 24, 10030-10039. https://doi.org/10.1364/OE.24.010030

[20] Synopsys (2012) Sentaurus TCAD.

https://www.synopsys.com/con-tent/dam/synopsys/silicon/datasheets/sentaurus_ds . $\mathrm{pdf}$ 